\title{
Nonlinear evolution of plasmoid structure
}

\author{
Shu A. Abe and M. Hoshino \\ University of Tokyo, 7-3-1 Hongo, Bunkyo, Tokyo 113-0033, Japan \\ (Received June 21, 2000; Revised January 26, 2001; Accepted February 23, 2001)
}

\begin{abstract}
The plasmoid observed in the Earth's magnetotail shows a wide variety of the complicated plasma structures that are not simply described by the standard Petschek reconnection model. The interaction of the plasmoid propagating tailward with the surrounding plasmas of the plasma sheet at rest may be important to understand the plasma sheet structure and the plasma heating observed in the magnetotail. The nonlinear time evolution of the plasmoid is studied by using a large-scale, high-resolution, two dimensional MHD simulation code. Several discontinuities/shocks are formed in association with magnetic reconnection: 1) a pair of the standard Petscheck-type slow shock waves emanating from the X-type neutral point, 2) the tangential discontinuity inside the plasmoid that separates the accelerated plasmas from the original plasma sheet plasmas, 3) the slow shock with a "crab-hand" structure surrounding the front-side of the plasmoid, 4) the intermediate shocks in the edge of the plasma sheet inside the plasmoid, and 5) the contact discontinuity inside the plasma sheet that separates the shock-heated plasmas from the Joule heated plasmas by the magnetic diffusion at the X-type neutral point. We also discuss how those plasma discontinuities/shocks structures are affected by the lobe/mantle plasma condition.
\end{abstract}

\section{Introduction}

The scenario of the plasmoid formation in the Earth's magnetotail was proposed by Hones (1977) based on the satellite observations. A large-scale magnetic island containing a hot and dense plasma is formed in association with the magnetic reconnection which is initiated in the nearEarth neutral line, and the magnetic island, so called plasmoid, is propagating tailward. Since the evolution of the plasmoid is considered to be produced by the magnetic reconnection process, the boundary structure of the plasmoid can be expected to be a slow-mode shock (Petschek, 1964). The test to confirm the structure of the plasma sheet has been performed by using the ISEE, AMPTE, and Geotail satellites' observations, and the existence of slow-mode shocks in the Earth's magnetotail has been confirmed (Feldman et al., 1987; Saito et al., 1995; Seon et al., 1996).

The plasmoid observed in the Earth's magnetotail, however, shows a wide variety of the complicated plasma structures that are not simply described by the standard Petschek reconnection model. For example, while the plasmoid is propagating tailward, the plasmoid compresses the surrounded region, which is observed as the traveling compression region (TCR) (Slavin et al., 1984). Hoshino et al. (2000) found that the two different plasma regions exist inside the plasma sheet during the magnetic reconnection in the magnetotail, and two boundaries are identified as a slowmode shock and a contact discontinuity that separates the slow shock heated plasmas from the isotropic plasmas in the plasma sheet. Terasawa et al. (1996) discussed the multielectric current structures in the plasma sheet, which sug-

Copy right (c) The Society of Geomagnetism and Earth, Planetary and Space Sciences (SGEPSS); The Seismological Society of Japan; The Volcanological Society of Japan; The Geodetic Society of Japan; The Japanese Society for Planetary Sciences. gests that another discontinuity is formed in addition to the standard slow shocks. Whang et al. (1997) reported that the rotational discontinuity is often attached to the slow shock in the magnetotail. We think that the nonlinear time evolution of the magnetic reconnection/plasmoid can produce such complicated structures.

During magnetic reconnection, the lobe plasmas are convected toward the plasma sheet, and are ejected outward from the X-type neutral region. Those accelerated plasmas collide with the original plasma sheet plasmas at rest before reconnection. The interaction between two plasma populations produces the compressed plasma region that contains the hot and high-density plasmas. The interface should be the tangential discontinuity that separates the accelerated plasmas from the original plasma sheet that exists already inside the plasma sheet before the onset of reconnection. Due to the interaction, MHD waves may be emitted from the plasmoid, and would produce a complicated structure in the plasma sheet.

To understand the dynamic structure of the plasma sheet, many people have studied the nonlinear time evolution of the plasma sheet by using MHD simulations (e.g., Ugai and Tsuda, 1977; Sato and Hayashi, 1979; Scholer and Roth, 1987; Otto et al., 1990; Birn and Hesse, 1991). The effect of the normal magnetic field $B_{Z}$ on the nonlinear evolution of the plasma sheet in the Earth's magnetotail geometry has been extensively studied by Hesse and Birn (1991). In this paper, we examine a long time evolution of the Harris-type plasma by using a high-resolution, two dimensional MHD simulation, and discuss the formation of multi-structure organized by the MHD waves/discontinuity in the plasma sheet. 
Table 1. Initial conditions of RUN 1-3: plasma density $\rho_{Z=0}$ and gas pressure $P_{Z=0}$ and the sound velocity $C_{S Z=0}$ at the neutral sheet, and plasma density $\rho_{Z=\infty}$ and gas pressure $P_{Z=\infty}$ and the Alfvén velocity in the lobe $V_{A Z=\infty}$.

\begin{tabular}{ccccccc}
\hline \multicolumn{7}{c}{ Initial parameters and phase velocity } \\
\hline RUN & $\rho_{Z=0}$ & $P_{Z=0}$ & $C_{S Z=0}$ & $\rho_{Z=\infty}$ & $P_{Z=\infty}$ & $V_{A Z=\infty}$ \\
\hline 1 & 1.0 & 0.625 & 1.12 & 1.0 & 0.125 & 1.0 \\
2 & 1.0 & 0.625 & 1.12 & 0.2 & 0.125 & 2.23 \\
3 & 1.0 & 0.625 & 1.12 & 5.0 & 0.125 & 0.45 \\
\hline
\end{tabular}

\section{Simulation Model}

\subsection{Basic equations}

In our simulation study, we used MHD equations below:

$$
\begin{aligned}
\frac{\partial}{\partial t} \rho & =-\nabla \cdot(\rho \mathbf{V}), \\
\rho \frac{d}{d t} \mathbf{V} & =-\nabla P+\mathbf{J} \times \mathbf{B}, \\
\frac{\partial}{\partial t} \mathbf{B} & =\nabla \times(\mathbf{V} \times \mathbf{B})+\eta \nabla^{2} \mathbf{B}-\nabla \eta \times \mathbf{J}, \\
\frac{\partial}{\partial t} P & =-\gamma P(\nabla \cdot \mathbf{V})-\mathbf{V} \cdot \nabla P+(\gamma-1) \eta J^{2},
\end{aligned}
$$

where $\mathbf{V}$ is the velocity vector, $\mathbf{B}$ is the magnetic field vector, $P$ is the gas pressure, and $\rho$ is the plasma density. We choose the ratio of the specific heat $\gamma$ as 2 in our twodimensional simulation.

The spatial scale is normalized by the thickness of the plasma sheet $\lambda$, and the velocity is normalized by the Alfvén velocity $V_{A}$, which is defined by $B_{Z=\infty} / \sqrt{\rho_{Z=0}}$ using the lobe magnetic field and the density in the plasma sheet. The time is normalized by the Alfvén transit time $\tau_{A}=\lambda / V_{A}$, and the magnetic field and the density are normalized by the lobe magnetic field and the density in the plasma sheet, respectively.

The resistivity $\eta$ is localized in space and is time-stationary in our simulation. The magnetic Reynolds number $\left(R_{M}\right)$ is 80 at the $\mathrm{X}$-point and $R_{M}=500$ in the other region far from the X-type region. The thickness of the localized resistivity is assumed to be as large as the plasma sheet thickness.

To simulate a large-scale plasmoid evolution, we took the size of the simulation system $L_{X} \times L_{Z}=280 \lambda \times 20 \lambda$. The number of grid points is set to be $8400 \times 600$ in $X$ and $Z$ to simulate correctly the nonlinear evolution of the plasmoid structure. In our code, the 4th order finite difference in space and the 4th order Runge-Kutta method in time are used.

\subsection{Initial conditions}

Our initial pressure equilibrium is assumed to be the Harris type configuration described below:

$$
\begin{aligned}
& B=B_{Z=\infty} \tanh Z \\
& P=\frac{B_{Z=\infty^{2}}}{2} \cosh ^{-2} Z+P_{Z=\infty}
\end{aligned}
$$

Due to the symmetry of reconnection, we only solve one quadrant of the system $X<0, Z>0$, and the localized resistivity $\eta$ is situated at the origin of $X=0$ and $Z=0$. For the outer boundary condition, we use the so-called "free boundary" by combining the Neumann-type condition, i.e.,

$$
\frac{\partial}{\partial n}=0
$$

and the artificial wave damping layer. We use 30 grids from the boundary as the wave-damping region.

In the modeling of the plasma sheet evolution, the lobe/mantle plasma condition is one of the important parameters, because in the near Earth's magnetotail, the lobe is very tenuous region of plasmas which density is about $10^{-2} \sim 10^{-1} \mathrm{~cm}^{-3}$, while in the distant magnetotail the mantle having a cold-dense plasma with $\sim 1 \mathrm{~cm}^{-3}$ is believed to contact with the plasma sheet (e.g., Gosling et al., 1984). The magnetic field intensity in the lobe decrease with the distance from the Earth. Therefore, the speed of the Alfvén waves propagating around the plasmoid decrease as it propagates downward (tailward). To understand the basic interaction between the high-speed plasma produced by the reconnection process and the original plasma sheet plasma at rest, we study the nonlinear time evolution of the plasmoid in three typical plasma parameters listed in Table 1. RUN 1 is the uniform density case, RUN 2 corresponds to the near-Earth magnetotail case where the lobe plasma density is very low, and RUN 3 may be one case for the distant magnetotail with the high-density mantle plasma.

\section{Plasmoid Formation: Region Identification}

Let us study first the "plasmoid" structures for RUN 1 with the uniform plasma condition at $t=0$. Figure 1 shows three snapshots of the electric current density $J_{Y}$ at $t=30$, 60 , and 90 . One can find that a pair of electric current layers is developed from the X-type neutral point that we identify later as the Petschek type slow mode shocks. As the time goes on, the current layers in the fore part of the plasmoid/magnetic island gradually curve toward the plasma sheet. The curved structure is also attributed to the slow mode shock formed by the expansion of the plasmoid/ magnetic island toward the lobe/mantle region. In the following sections, we will argue several important regions one by one.

\subsection{Near X-point region: $0>X>-20: t=120$}

We focus first on the plasma sheet structure in the vicinity of the X-type neutral point. Figure 2 shows the snapshot of the plasma temperature at $t=120$. By checking the MHD Rankine-Hugoniot relations, we identify the boundary between the lobe and the hot plasma sheet as the slow-mode shock, and the hot plasma is mainly heated by the Petschektype reconnection.

Looking into the hot plasma sheet in detail, however, we find that hotter and thinner plasma layer is embedded in the plasma sheet. The hot plasma region (green color) is formed in the plasma sheet, and we find that the plasma is compressed in the hot plasma sheet. We think that the interface 


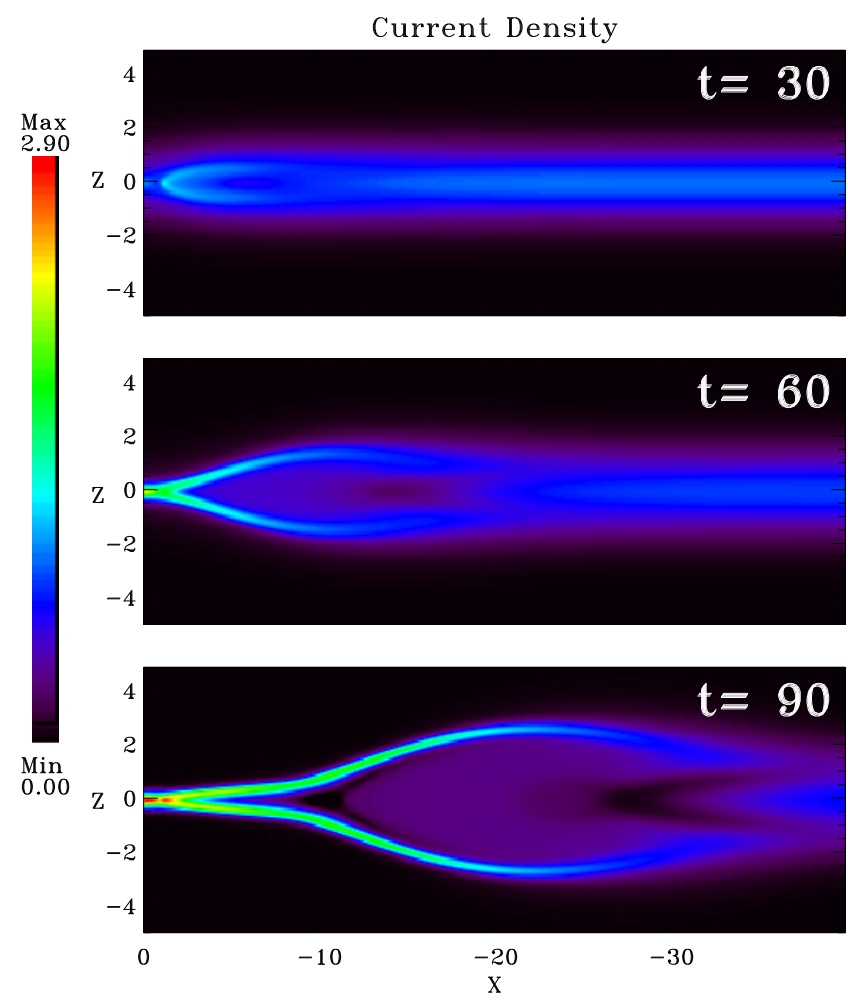

Fig. 1. Time evolution of the electric current structures during the early nonlinear stage of reconnection $t=30,60$, and 90: Current layer is split into a pair of thin layers at $t=90$, these layers gradually bend around $X=10$.

between the outer and inner plasma sheet is a contact discontinuity, because the plasma flow is almost along the $\mathrm{X}$ axis and does not cross the interface. The thin and inner hot plasma region is thought to be produced by Joule-heating around the X-type neutral point.

The similar plasma structure has been observed by the Geotail satellite, and the inner and outer plasma sheet structure has been discussed by Hoshino et al. (2000). They discussed that two discontinuities exist during magnetic reconnection in the magnetotail: one is the slow-mode shock, and the other is the contact discontinuity which separates the slow shock heated plasmas from the isotropic plasmas in the inner plasma sheet.

\subsection{Inner plasmoid structure: $-30>X>-60$ : $t=$ 140}

Next, we examine the inner structures of the magnetic island surrounded by the enhanced electric current layer. The top panel of Fig. 3 shows the color contour plot of the plasma gas pressure in the region from $X=-20$ to -70 at $t=140$, and the magnetic field lines are superimposed onto the color contour. The bottom panel displays the slice of $B_{Z}$ along the $X$-axis. We can find that the gas pressure and the $Z$-component of the magnetic field are simultaneously enhanced inside the plasmoid/magnetic island around $X \sim-50$. The plasma parameters change gradually across $X \sim-50$, and the boundary is not sharp, but it can be identified as the tangential discontinuity. The region $X>-50$ is occupied with the accelerated plasma by reconnection that were the lobe plasma before reconnection.

In order to understand the property of the plasma com-

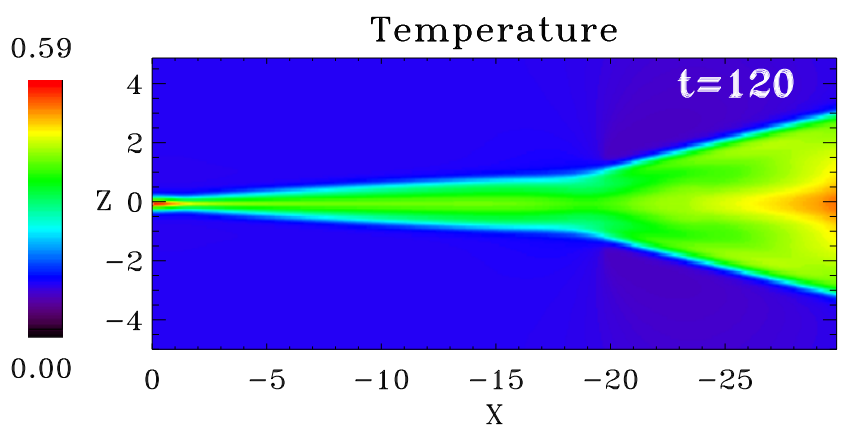

Fig. 2. Fine structure of the plasma temperature in the downstream region of the slow shock. This hot plasma region is embedded in the plasma sheet.

pression in $X$-direction, we study one dimensional shock tube problem that mimics the interaction of the accelerated, high-speed plasma flow by reconnection with the plasma sheet plasmas at rest. In our shock tube modeling, the lefthand side region is assumed to be the high-speed plasma flow region by the reconnection outflow, and the right-hand region corresponds to the original plasma sheet at rest. The left three panels in Fig. 4 show the initial state of a discontinuity: from top, the plasma flow velocity, the gas pressure, the magnetic field $B_{Z}$, and the plasma density. As the time goes on, the initial discontinuity develops into one pair of the fast-mode shocks and a tangential discontinuity. Due to the compression of high-speed plasma flow ejected from the left-hand boundary, the magnetic fields are intensified behind the tangential discontinuity (we call this region as Region I). In Region I, the plasmas are occupied by the accelerated plasma originated from the lobe, while in front of the tangential discontinuity the plasmas belong to the plasma sheet plasma existing before the reconnection onset (we call this region as Region II). In Region II, the plasma is compressed, but no $B_{Z}$ magnetic field exists. The above numerical Riemann solution can be also analytically solved, and we obtained the consistent result.

The shock tube solution can be compared with the simulaton result in Fig. 4. Tangential discontinuity is formed at $X \sim-50$ and almost no $B_{Z}$ magnetic field exist in forward-side of the plasmoid/magnetic island in the neutral line.

Since the propagation speed of the tangential discontinuity is slower than the speed of the reconnection outflow ejected from the X-type region, the reconnecting magnetic field lines should be piled up behind the tangential discontinuity. If the outflow exceeds the fast-mode speed, another discontinuity, as the fast reversed shock, should be formed between the reconnection outflow region and the compressed plasma region behind the tangential discontinuity. From the magnetic field plot in Fig. 3, we can find the reverse shock around $X \sim-32$ as the boundary of the pile up magnetic field. Whether or not the outflow speed exceeds the fast mode speed depends on the lobe plasma situation. According to the standard reconnection model, the outflow velocity becomes almost the Alfvén velocity of $B_{Z=\infty} / \sqrt{\rho_{Z=\infty}}$ in a strong slow shock limit. The lobe magnetic pressure balances with the gas pressure in the neu- 

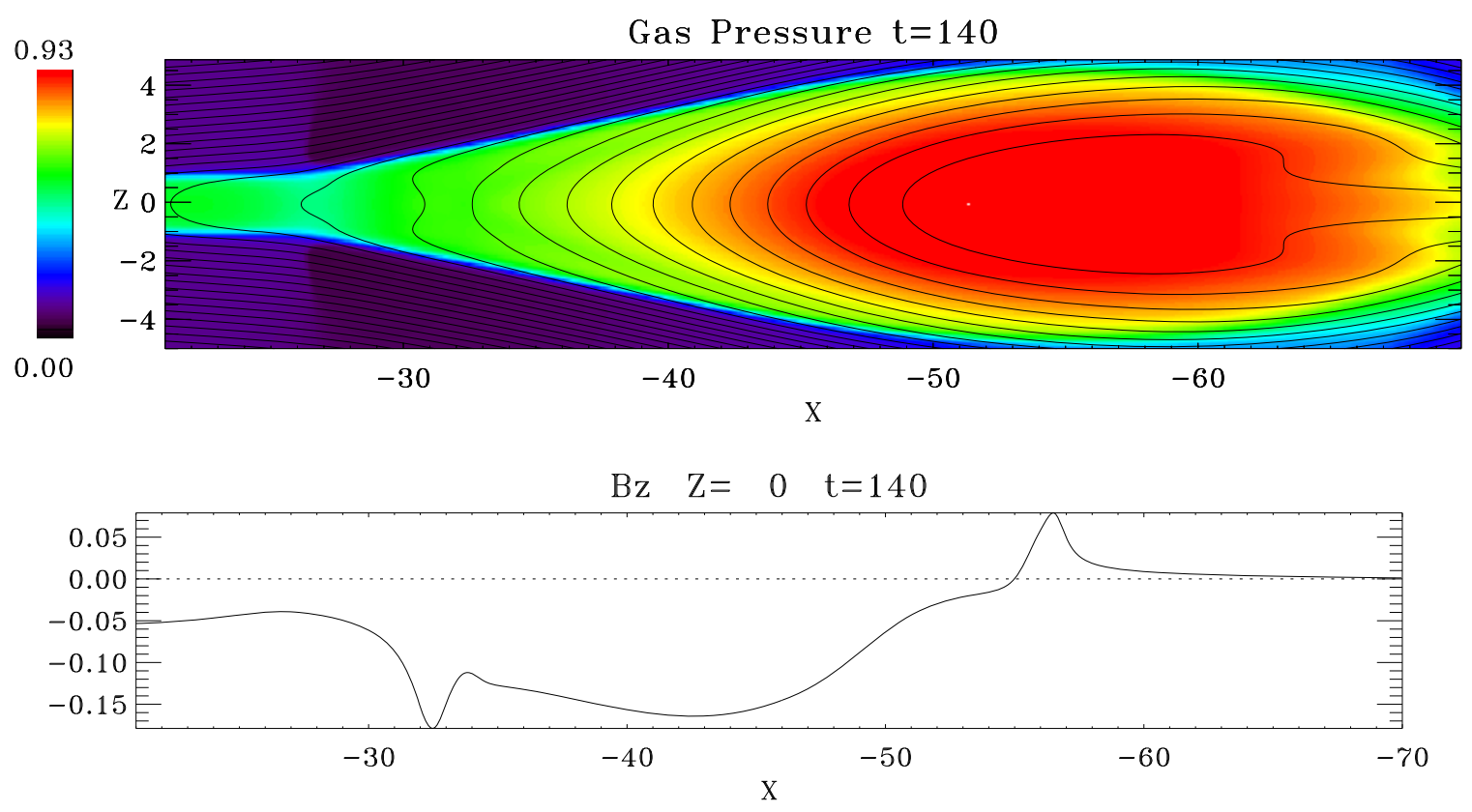

Fig. 3. Plasmoid Structure at $t=140$ : Top panel shows the gas pressure and the magnetic field lines around the plasmoid. Bottom panel shows the profile of $B_{Z}$ on the neutral line. This reconnecting magnetic field lines accumurate into the plasmoid, and the high gas pressure region is formed behind the accumurated magnetic field region. This boundary is identified as the tangential discontinuity.
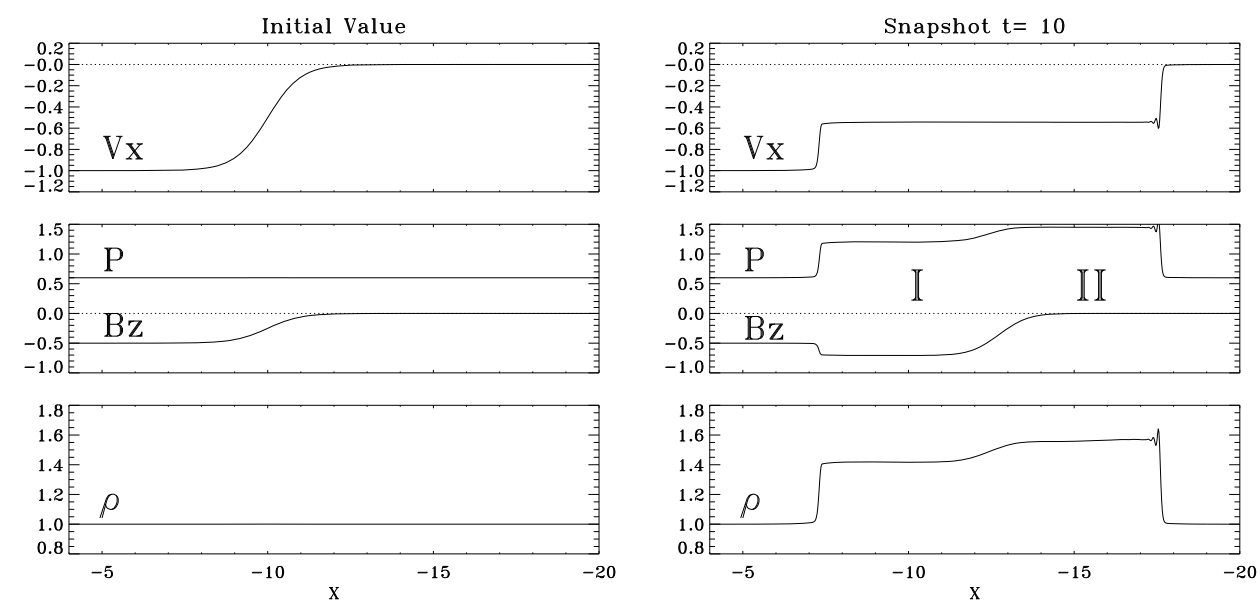

Fig. 4. A shock tube problem that mimics the plasmoid inner structure along the neutral line: Left-hand panel shows the initial conditions and right-hand panel is the snapshot at $t=10$. These initial discontinuities are separated into three MHD discontinuities. We can observe that the tangential discontinuity at $X=-12$ and a pair of fast mode shocks are formed. Region I corresponds to the left-hand side region of the tangential discontinuity and there intensified magnetic field.

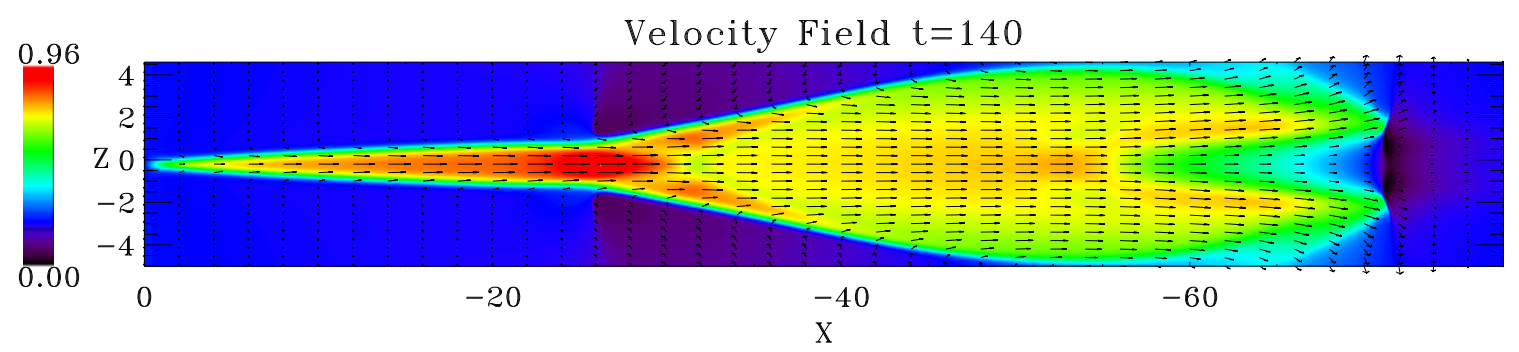

Fig. 5. Plasma flow pattern at $t=140$. 


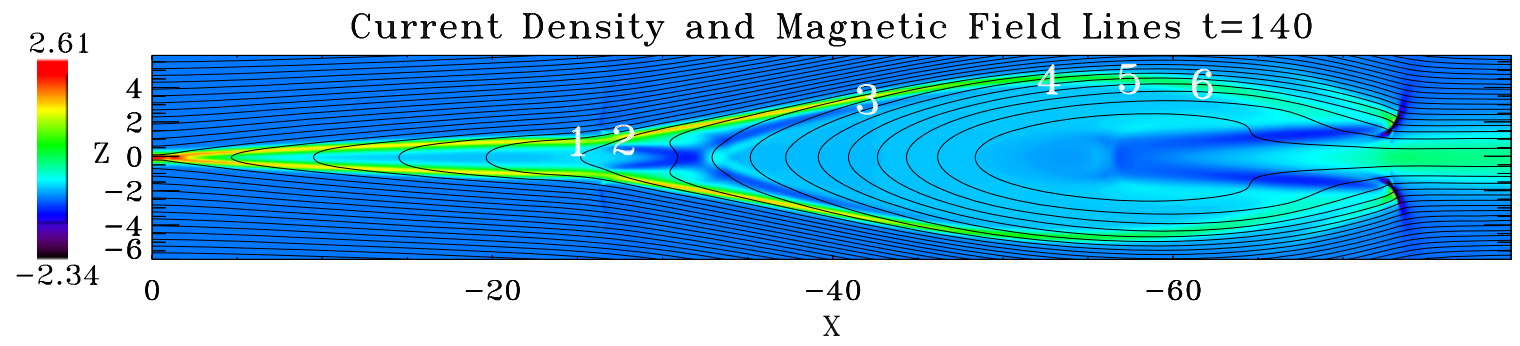

Fig. 6. Electric current and magnetic field lines at $t=140$. The slow-mode Rankine-Hugoniot conditions are checked for the regions denoted by the number 1 to 6 .

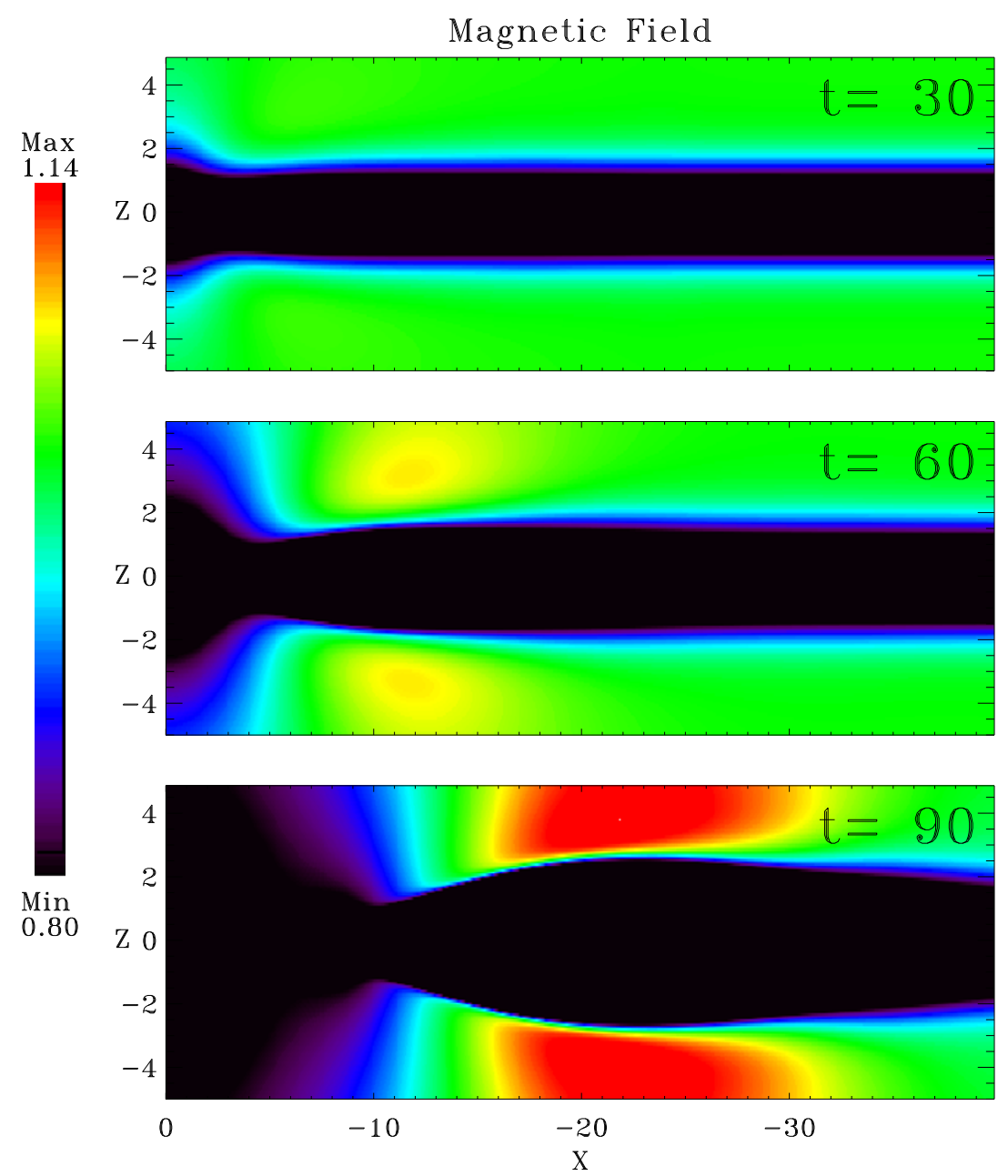

Fig. 7. Magetic field strength enhances in the lobe in association with the plasmoid formation.

tral sheet, then $\rho_{Z=0} T_{Z=0}=P_{Z=0}=B_{Z=\infty}^{2} / 2$. Therefore, the Mach number $\mathcal{M}$ of the outflow plasma is $\mathcal{M}^{2}=$ $(2 / \gamma)\left(\rho_{Z=0} / \rho_{Z=\infty}\right)$ where we assume that the magnetic field is weak in the outflow region. Although this is a very rough estimation, we could expect the formation of the reverse shock in the outflow region when the lobe density is smaller than the plasma sheet density.

Figure 5 shows the contour of the $V_{X}$ and the flow vector at $t=140$. The sharp discontinuity of the plasma velocity is formed at around $X=-30$, and we can also observe the kink of the plasmoid/magnetic island boundary in the contour plot of $V_{X}$ around $X=-25$. We think that this region is produced due to the fast-mode compressional waves propagating backward from the plasmoid/magnetic island, and that both the discontinuity of velocity and the kink also correspond to the position of the fast mode wavefront (FWF) expected in the shock tube modeling.

The kink position in the current layer $X=-25$ in Fig. 5 is not same as the pile up magnetic field boundary $X=-30$ in Fig. 3, because the outflow plasma is not uniform in the $Z$-direction. The Mach number in the central region confined by the contact discontinuity is slower than that in the 

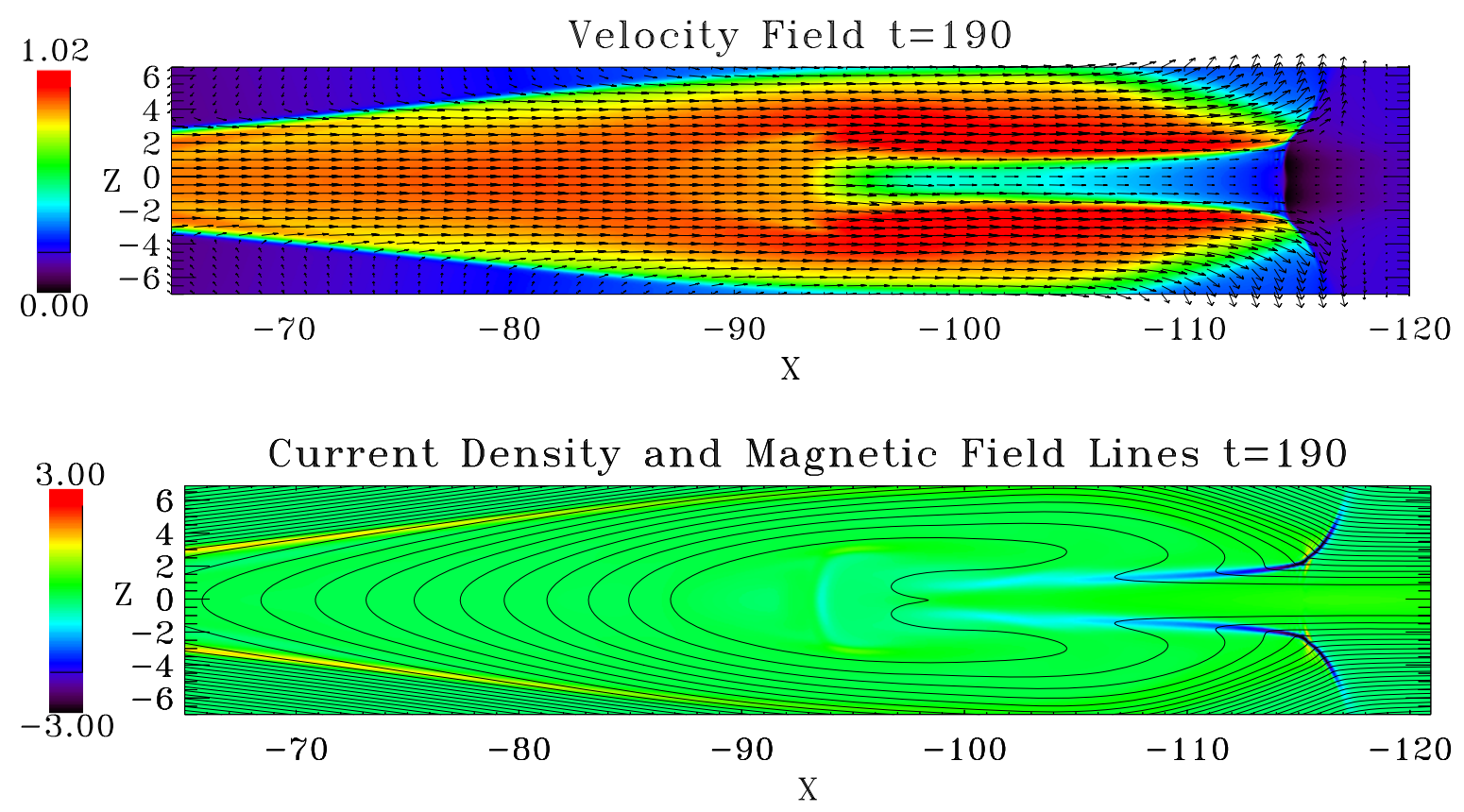

Fig. 8. Magnetic-field lines show drastic kink at the intermediate shock at $t=190$.

region sandwiched between the slow shock and the contact discontinuity. Therefore, the reverse shock front starts to bend, and forms a concave shape front.

The forward of the compressional FWF which is expected in shock tube situation, however, can hardly be observed in Fig. 5. Since the plasma flow in two dimensional system expands into the lobe region, and the amplitude of the compressional FWF becomes weak as it propagates. We must take into account the effect of plasma expansion to get better modeling of the fast mode wave properties.

3.3 Plasmoid boundary region: $X>-70: t=140$

We discuss here the intensified and curved electric current layer surrounding the plasmoid/magnetic island. It is known that one pair of the slow-mode shocks is formed during magnetic reconnection in Petschek steady-state model, and we can confirm that the slow shock pair is formed around the $\mathrm{X}$-type region in our simulation result. The enhanced electric current layer extends further downstream in such a way that the current layer surrounds the plasmoid/magnetic island. We check whether or not every each point on this current layer satisfies the slow shock jump conditions, and we confirm that the boundary between the plasmoid/magnetic island and the lobe is the slow-mode shock. The numbers indicated in Fig. 6 show the positions that we examine the slow shock Ranikine-Hugoniot relations. The Mach number, the shock angle between the shock normal and the magnetic field, the plasma beta in upper-region of each region are listed in Table 2.

The slow-mode Mach number along the slow shock from the X-type neutral point to the kink position around $X=$ -25 is about 1.85 , which is almost constant. However, across the kink structure of the plasmoid/magnetic island, we find that the slow mode Mach number $\mathcal{M}_{1}$ changes discontinuously from 1.85 (position 1) to 2.34 (position 2).
Table 2. Identification of the slow shock: $X$ and $Z$ show the positions that we examine the Rankine-Hugoniot relations, Mach number $\mathcal{M}_{1}$, the angle between the shock normal and the magnetic field $\theta_{B N 1}$, the plasma beta $\beta_{1}$ in the shock upstream region.

\begin{tabular}{cccccc}
\hline \multicolumn{7}{c}{ Slow shock } \\
\hline No. & $X$ & $Z$ & $\mathcal{M}_{1}$ & $\theta_{B N 1}$ & $\beta_{1}$ \\
\hline 1 & -24.2 & 0.93 & 1.85 & 87.4 & 0.18 \\
2 & -26.9 & 1.06 & 2.34 & 88.6 & 0.10 \\
3 & -41.2 & 3.42 & 2.28 & 86.5 & 0.07 \\
4 & -51.9 & 4.60 & 1.81 & 87.4 & 0.13 \\
5 & -56.6 & 4.60 & 1.50 & 87.2 & 0.26 \\
6 & -60.9 & 4.31 & 1.30 & 87.7 & 0.39 \\
\hline
\end{tabular}

As further going downward along the slow shock layer, the slow Mach number $\mathcal{M}_{1}$ in the upstream region gradually decreases. Beyond the position of the number 6 , the slow shock identification becomes difficult because $\mathcal{M}_{1}$ becomes close to unity.

The slow shock region from the X-type neutral point to the position 4 is basically formed by the super slow convection flow toward the plasma sheet, while the front side region beyond the position 4 is formed by the expanding/growing nature of the plasmoid/magnetic island in time and in space. The size of the plasmoid/magnetic island becomes larger and larger as the time goes on. In Fig. 7, we can find the magnetic field is enhanced in the outer region of the plasmoid boundary, corresponding to the observed signature of TCRs in the lobe (Slavin et al., 1984). It should be also noted that the front side slow shock has been identified by the Geotail observation (Saito et al., 1995). 


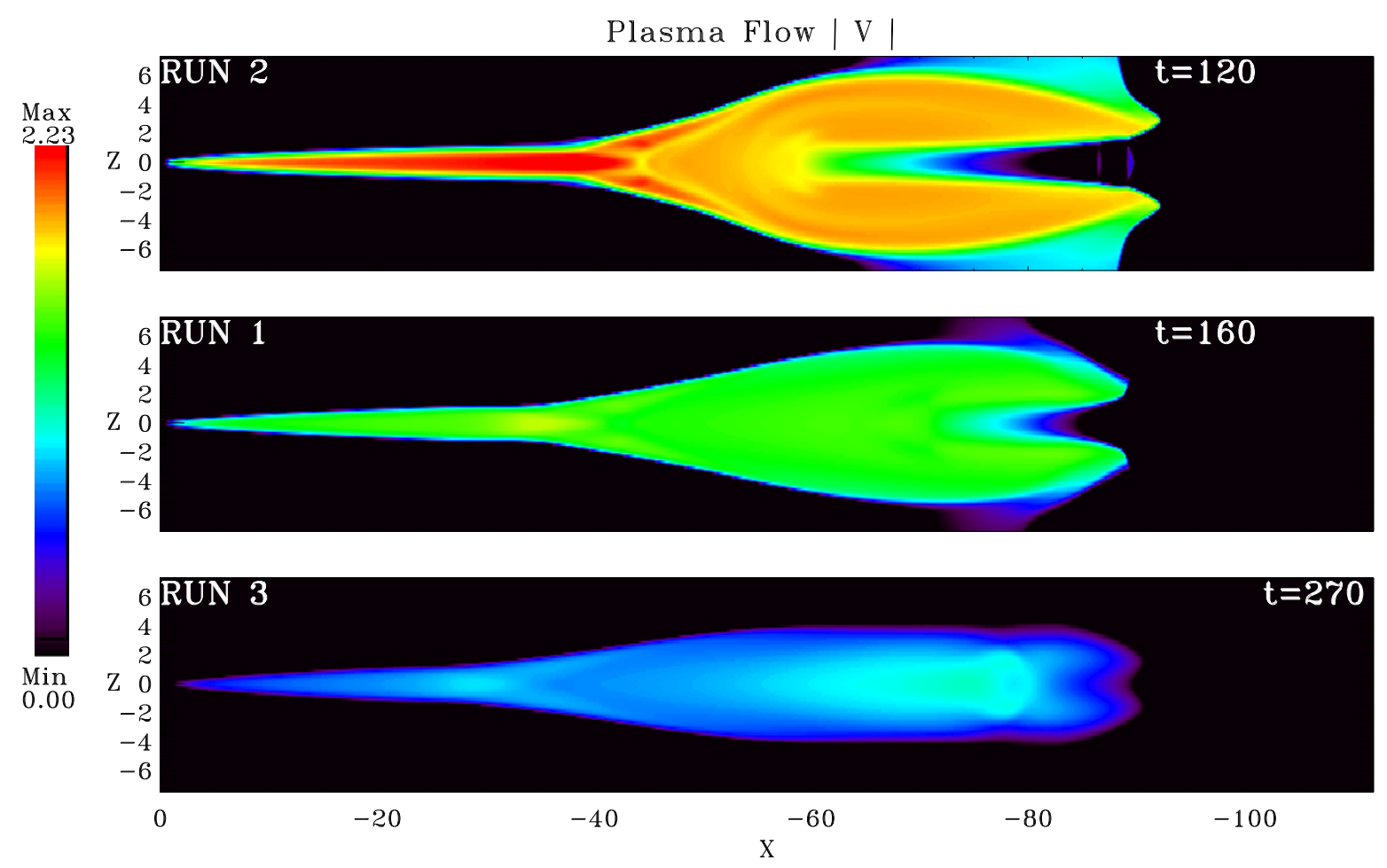

Fig. 9. Dependence on the initial lobe density of the plasmoid structure: Top RUN $2\left(V_{\max } \sim 2.23\right)$, Center RUN 1 ( $\left.V_{\max } \sim 1.00\right)$, Bottom RUN 3 $\left(V_{\max } \sim 0.46\right)$, color bar is log-scaled.

3.4 Forward-side structure of the plasmoid: $-105>$ $X>-115: t=190$

The top panel of the Fig. 8 shows $V_{X}$ superimposed with the plasma flow vector at $t=190$, and the bottom shows the strength of the current density superimposed with the magnetic field lines. On the top figure, one can observe that the plasma flow velocity near the neutral sheet is much slower than the outer plasma flow from $X=-95$ to -115 . It seems that the outflow plasma cannot enter into the center of the plasmoid/magnetic island, because the plasma sheet consists of the high density plasma with a large inertia.

In the region between the slow flow and the fast flow in the plasma sheet on the front-side of the plasmoid/magnetic island $X=-95 \sim-115$, we find another shock that the magnetic field reverse its tangential component. The region corresponds to the blue color region on the current density plot in Fig. 8. By checking the Rankine-Hugoniot relations across the boundary, we can identify the region as the intermediate shock, (e.g., Wu, 1987; Hau and Sonnerup, 1989). The upstream region is the forward region of the plasmoid/magnetic island where the plasma flow is slow. The wave-front of the intermediate shock is thought to correspond to the inner edge of the "crab-hand" shaped plas$\mathrm{moid} / \mathrm{magnetic}$ island.

\section{Dependence on Lobe Density Profile}

We analyzed the simulation result RUN 1 in the previous section and understood the formation process of several important structures embeded in the plasmoid/magnetic island. In this section, we discuss how the plasmoid evolution is modified by the lobe plasma condition (see Table 1). We compare three simulation RUN 1, 2, and 3 in Fig. 9.
From the top to the bottom, RUN 2 with the low plasma density case at $t=0$, RUN 1 with the uniform plasma density case, and RUN 3 with the high-density plasma in the mantle/lobe are shown. Note that the plasma sheet conditions are same for RUN 1, 2, and 3. Each snapshot is taken when the front-face of the plasmoid/magnetic island reaches almost the same X-position in the simulation box.

The region behind the tangential discontinuity is occupied with the hotter and denser plasmas than the lobe plasmas, except for the vicinity of the neutral line. We can find that plasmas are accelerated to Alfvén velocity defined in the lobe, and the speed of plasmoid/magnetic island propagation is about a half of the Alfvén velocity. The outside boundary surrounding the plasmoid/magnetic island is curved into the plasma sheet, and we also confirm that these boundaries are the slow-mode shock. These properties are common among RUN 1, 2, and 3. Around the front region of the plasmoid/magnetic island, however, the plasmoid expansion into the lobe is different, and the "crab-hand" shaped structure is no longer observed in the high-density lobe case of RUN 3.

The outer plasmoid/magnetic island boundary is also identified as the slow-mode shock for RUN 1, 2, and 3. It is interesting to note that the slow-mode shock can be formed even for the high density lobe case of RUN 3. As the time goes on, the original plasma sheet plasma is ejected from the X-point region, and the plasma is supplied from the lobe. During this stage, the compressed plasma in association with the slow-mode shock can be formed. On the other hand, the front-side region of the plasmoid/magnetic island is occupied with the low-density plasmas that are originally in the plasma sheet. Although the plasma in this region is com- 
pressed due to the reconnection outflow, but the density is not necessarily higher than the lobe plasma density. Therefore, we think that if the lobe plasma is denser and colder than the plasma sheet, the compressed region with hot and low-density plasma may be formed in the front-side of the plasmoid/magnetic island. According to the plasmoid observations so far, the plasmoids are characterized by the hot and teneous plasmas (e.g., Machida et al., 1994; Ieda et al., 1998). We think that our study RUN 3 is consistent with the observation in the magnetotail.

Let us study the opening angle of the slow-mode shocks and the inner-edge structure of the plasmoid/magnetic island. In the low lobe density case of RUN 2, the plas$\mathrm{moid} / \mathrm{magnetic}$ island can expand wider toward the lobe, and the reconnection outflow plasma can hardly penetrate into the center of the plasmoid/magnetic island. The plasma divergence causes the deformation of magnetic field topology of inner-edge of the plasmoid/magnetic island, and the intermediate shocks can develop. This behavior is well observed in the low plasma density case.

In case of the high-density lobe for RUN 3 as shown in Fig. 9, the crab-hand shape is not well developed, and the intermediate shock no longer appears inside the plasmoid/ magnetic island. However, in front of the plasmoid/magnetic island $X \sim-90$, we can find the bow shock type structure produced by the tailward propagation of plasmoid/magnetic island. This front boundary has the intermediate shock behavior because the magnetic field polarity is changed and because the plasma is compressed.

\section{Summary}

We have studied the nonlinear evolution of plasmoid/ magnetic island by using the high-resolution, 2-D MHD simulation code, and have discussed how the lobe/mantle plasma affects on the plasmoid/magnetic island dynamic structure.

Once the reconnection triggered by the localized resistivity on an X-type neutral point, the Petschek-type slow-mode shock is formed, and the fast-mode rarefaction wave propagates toward the lobe in its upstream region. The lobe plasmas are convected down to the plasma sheet, and the plasmas can be heated by the slow shock. Further downstream, we find the higher temperature regions where the plasmas are heated by the magnetic diffusion process around the Xtype neutral point. The interface between the shock heated plasma and the Joule-heated plasma sheet can be recognized as the contact discontinuity. These outflow plasmas are accelerated to the nearly Alfvén velocity defined in the lobe, and they collide with the original plasmas at rest and compress the surrounding medium. Therefore, the tangential discontinuity is formed so as to separate the original plasma sheet plasmas from the reconnection accelerated plasmas, and the magnetic field starts to pile up behind the tangential discontinuity. The structure inside the compressed region depends on the lobe plasma condition. In case of the low lobe density, the "crab-hand" shaped structure is formed in the front region of the plasmoid/magnetic island, and the interface develops into the intermediate shock.

In summary, five kinds of structures could be observed in plasmoid/magnetic island: 1) the Petschek-type slow-mode

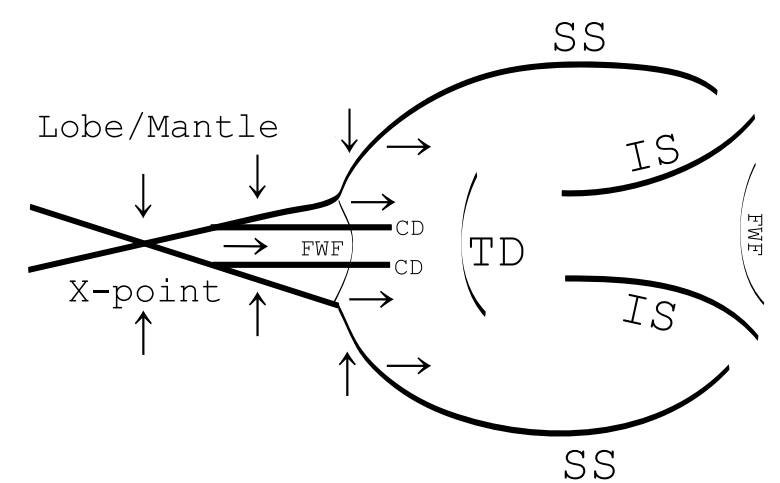

Fig. 10. Summary of our results.

shocks around the reconnection region, 2) the Joule heated plasmas region inside the contact discontinuities downstream the slow-mode shocks, 3) the tangential discontinuity inside the plasmoid, 4) the expanding slow shocks which propagate downward, 5) the intermediate shocks formed in front-side of the plasmoid. The above structures are summarized in Fig. 10.

\section{References}

Birn, J. and M. Hesse, MHD simulations of magnetic reconnection in a skewed three-dimensional tail configuration, J. Geophys. Res., 96, 2334, 1991.

Feldman, W. C., R. L. Tokar, J. Birn, E. W. Hones, Jr., S. J. Bame, and C. T. Russell, Structure of a slow mode shock observed in the plasma sheet boundary layer, J. Geophys. Res., 92, 83-94, 1987.

Gosling, J. T., D. N. Baker, S. J. Bame, E. W. Hones, Jr., D. J. McComas, R. D. Zwickl, J. A. Slavin, E. J. Smith, and B. T. Tsurutani, Plasma entry into the distant tail lobes: ISEE-3, Geophys. Res. Lett., 11, 1078-1081, 1984.

Hau, L. N. and B. U. O. Sonnerup, On the structure of resistive MHD intermediate shocks, J. Geophys. Res., 94, 6539-6551, 1989.

Hesse, M. and J. Birn, On dipolarization and its relation to the substorm surrent wedge, J. Geophys. Res., 96, 19,417-19,426, 1991.

Hones, E. W., Jr., Substorm processes in the magnetotail: Comments on 'On hot tenous plasma, fireballs, and boundary layers in the Earth's magnetotail' by L. A. Frank, K. L. Ackerson, and R. P. Lepping, J. Geophys. Res., 82, 5633, 1977.

Hoshino, M., T. Mukai, I. Shinohara, Y. Saito, and S. Kokubun, Slow shock downstream structure in the magnetotail, J. Geophys. Res., 105, $337-$ $347,2000$.

Ieda, A., S. Machida, T. Mukai, Y. Saito, T. Yamamoto, A. Nishida, T. Terasawa, and S. Kokubun, Statistical analysis of the plasmoid evolution with Geotail observations, J. Geophys. Res., 103, 4453-4465, 1998.

Machida, S., T. Mukai, Y. Saito, T. Obara, T. Yamamoto, A. Nishida, M. Hirahara, T. Terasawa, and S. Kokubun, GEOTAIL low energy partical and magnetic field observations of a plasmoid at $X_{G S M}=-142 R_{E}$, Geophys. Res. Lett., 21, 2995-2998, 1994.

Otto, A., K. Schindler, and J. Birn, Quantitative study of the nonlinear formation and acceleration of plasmoids in the Earth's magnetotail, J. Geophys. Res., 95, 15,023-15,037, 1990.

Petschek, H. E., Magnetic field annihilation, NASA Spac. Publ., 425-439, 1964.

Saito, Y., T. Mukai, T. Terasawa, A. Nishida, S. Machida, M. Hirahara, K. Maezawa, S. Kokubun, and T. Yamamoto, Slow-mode shocks in the magnetotail, J. Geophys. Res., 100, 23,567-23,581, 1995.

Sato, T. and T. Hayashi, Externally driven magnetic reconnection and powerful magnetic energy converter, Phys. Fluid, 22, 1189-1202, 1979.

Scholer, M. and D. Roth, A simulation study on reconnection and smallscale plasmoid formation, J. Geophys. Res., 92, 3223-3233, 1987.

Seon, J., L. A. Frank, W. R. Paterson, J. D. Scudder, F. V. Coroniti, S. Kokubun, and T. Yamamoto, Observations of slow-mode shocks in Earth's distant magnetotail with the Geotail spacecraft, J. Geophys. Res., 101, 27,383-27,398, 1996. 
Slavin, J. A., E. J. Smith, B. T. Tsurutani, D. G. Sibeck, H. J. Singer, D. N. Baker, J. T. Gosling, E. W. Hones, and F. L. Scarf, Substorm-associated traveling compression regions in the distant tail: ISEE 3 observations, Geophys. Res. Lett., 11, 657, 1984.

Terasawa, T., H. Kawano, I. Shinohara, T. Mukai, Y. Saito, M. Hoshino, A Nishida, S. Machida, T. Nagai, T. Yamamoto, and S. Kokubun, On the determination of a moving MHD structure: Minimization of the residue of integrated Faraday's equation, J. Geomag. Geoelectr., 48, 603-614, 1996.

Ugai, M. and T. Tsuda, Magnetic field-line reconnexion by localized en- hancement of resistivity. Part 1. Evolution in a compressible MHD fluid, J. Plasma Phys., 17, 337-356, 1977.

Whang, Y. C., D. Fairfield, E. J. Smith, R. P. Lepping, S. Kokubun, and Y. Saito, Observations of double discontinuities in the magnetotail, Geophys. Res. Lett., 24, 3153-3156, 1997.

Wu, C. C., On MHD intermediate shocks, Geophys. Res. Lett., 14, 668671, 1987.

S. A. Abe (e-mail: shuei@space.eps.s.u-tokyo.ac.jp) and M. Hoshino 